Article

\title{
Late time acceleration of the universe from quantum gravity
}

\author{
Xiankai PANG ${ }^{1}$ (1)
}

1 Arnold-Sommerfeld-Center for Theoretical Physics, Ludwig-Maximilians-Universität, Theresienstraße 37, Müchen, 80333, Germany; Xiankai.Pang@physik.uni-muenchen.de

\begin{abstract}
We show that the accelerating expansion phase of the universe can emerge from the group field theory formalism, a candidate theory of quantum gravity. The cosmological evolution can be extracted from condensate states using mean field approximation, in a form of modified FLRW equations. By introducing an effective equation of state $w$, we can reveal the relevant features of the evolution, and show that with proper choice of parameters, $w$ will approach to -1 , corresponds to the behaviour of cosmological constant, results in a late time acceleration and leads to de Sitter spacetime asymptotically.
\end{abstract}

Keywords: dark energy theory; quantum cosmology; quantum gravity phenomenology

\section{Introduction}

Since its discovery [1,2], the accelerating expansion of our universe remains a challenge to theoretical physics. One way to get the acceleration is to introduce dark energy, whose equation of state is close to -1 [3]. The simplest candidate would be the cosmological constant $\Lambda$ [4], but the smallness of its observed value [5], compared to the theoretical expectations from vacuum energy of particle physics [6] or from the renormalization flow of couplings in general relativity (GR) [7], making it difficult to find a compelling origin of $\Lambda$.

Another route, besides the more particle-theoretic approach, is to modify gravity theory [3]. In particular, the quantum gravity effects can be viewed as modifications to GR at the effective level. For example, the acceleration of universe can be reproduced within the formalism of asymptotically safe cosmology [8], of Dvali-Gabadadze-Porrati braneworld model [9], and of condensate cosmology in group field theory (GFT) [10], without the need of dark energy or cosmological constant. On the other hand, even if some dark energy fields do exist, their behaviour may subject to the quantum gravity effects, for instance some future singularities could be avoided [11].

In this work we also follow this approach, and show that in GFT condensate cosmology, the late time acceleration of the universe expansion will emerge naturally [10], without introducing any dark energy field which may have mysterious properties.

As a candidate theory of quantum gravity, instead of the familiar spacetime degrees of freedom, GFT provides more abstract, non-spatiotemporal entities, from which the continuum spacetime should emerge [12-14]. Furthermore, the cosmological evolution can be extracted from suitably constructed condensate states, which includes a large number of quantas to recover the continuum limit [15-22]. In this formalism the universe can be viewed as a quantum fluid constituted by GFT quanta, and its dynamics follows from the fundamental GFT theory through hydrodynamic approximation. Various works had been done in this direction, for example, using GFT condensate one can derive the modified FLRW equation [16], a varying Newton constant G [23], and the inflationary phase without the need of inflaton [24]. In this report we will summarize the main results obtained recently in [10], where we made a further step and showed that using GFT condensate it's also possible to reproduce a long lasting acceleration phase in our cosmological expansion at late times. 
The report is organized as follows. In section 2 we briefly review the basics of GFT formalism and its relation to cosmology. The dynamics and the modified FLRW equation are given in 3 , where we also introduce the effective equation of state to make our discussion more explicit. Section 4 presents our main result, that a long lasting accelerated expansion at late time can be reproduced in our model, of purely quantum gravity origin. Finally in section 5 we give a short summary of our results.

\section{Condensate cosmology in GFT}

For the emergence of $4 d$ spacetime, one usually chooses the GFT field to be a map over four copies of $S U(2)$ group, $\varphi: S U(2)^{4} \rightarrow \mathbb{C}, \varphi\left(g_{v}\right)=\varphi\left(g_{1}, g_{2}, g_{3}, g_{4}\right)$ [12]. And geometrically to associate the basic quanta of our theory with a tetrahedra, we further require that the field $\varphi\left(g_{v}\right)$ to be right invariant $\varphi\left(g_{v} h\right)=\varphi\left(g_{1} h, g_{2} h, g_{3} h, g_{4} h\right)=$ $\varphi\left(g_{v}\right), \forall h \in S U(2)$ [12]. Furthermore, since the spacetime is emergent, especially there is no time to start with, one usually needs a free massless scalar field as a relational clock to track the evolution, this way the field $\varphi\left(g_{v}, \phi\right)$ becomes time dependent.

The right invariance of $\varphi\left(g_{v}, \phi\right)$ under $S U(2)$ enables us to project the field using Peter-Weyl decomposition onto the complete and orthonormal basis of $L^{2}\left(S U(2)^{4} / S U(2)\right)$, the space of square integrable functions over quotient space $S U(2)^{4} / S U(2)$. Such basis can be given by the spin network vertex functions $\kappa_{\vec{x}}\left(g_{v}\right)$, which associates graphically to a spin network 4 -vertex labelled by $\vec{x}=(\vec{j}, \vec{m}, \iota)$, i.e. a node with $d=4$ open links associated with 4 spins $\vec{j}=\left(j_{1}, j_{2}, j_{3}, j_{4}\right)$, together with angular momentum projections $\vec{m}$, and the intertwiner quantum number $\iota$ associated instead to the node itself [25]. One thing to be noted is that these quantum numbers characterize geometrical properties of the tetrahedra, and in particular each spin label $j_{i}$ determines area of the associated face $[25,26]$.

More explicitly, in the second quantized form, the field operators can be written as

$$
\hat{\varphi}\left(g_{v}, \phi\right)=\sum_{\vec{x}} \hat{c}_{\vec{x}}(\phi) \kappa_{\vec{x}}\left(g_{v}\right), \hat{\varphi}^{\dagger}\left(g_{v}, \phi\right)=\sum_{\vec{x}} \hat{c}_{\vec{x}}^{\dagger}(\phi) \bar{\kappa}_{\vec{x}}\left(g_{v}\right)
$$

where $\hat{c}_{\vec{x}}(\phi)$ and $\hat{c}_{\vec{x}}^{\dagger}(\phi)$ are annihilation and creation operator respectively.

Having the basic operators in hand, the next step would be to find a suitable state for the interested spacetime, at least approximately. In our case, the homogeneous universe at a given time can be approximated by the coherent peaked state (CPS), which is constituted by a large number of quantas concentrated in a fixed relational time $\phi_{0}$ $[16,22]$

$$
\left|\sigma_{\varepsilon} ; \phi_{0}, \pi_{0}\right\rangle=\mathcal{N}(\sigma) \exp \left(\int(\mathrm{d} g)^{4} \mathrm{~d} \phi \eta_{\varepsilon}\left(\phi-\phi_{0}, \pi_{0}\right) \tilde{\sigma}\left(g_{v}, \phi\right) \hat{\varphi}^{\dagger}\left(g_{v}, \phi\right)\right)|0\rangle,
$$

with $|0\rangle$ is vacuum state, defined by $\hat{\varphi}\left(g_{v}, \phi\right)|0\rangle=0$ for all $g_{v}$ and $\phi$, and $\mathcal{N}(\sigma)$ is a suitable normalization factor. Peaking function $\eta_{\varepsilon}\left(\phi-\phi_{0}, \pi_{0}\right)$ is peaked around $\phi_{0}$ with a typical width given by $\varepsilon$, and the fluctuations of the operator corresponding to the conjugate momentum of the scalar field $\phi$ are controlled by $\pi_{0}$. We call $\tilde{\sigma}\left(g_{v}, \phi\right)$ the reduced condensate wave function and assume that it does not modify the peaking property of the CPS [22].

The wave function $\tilde{\sigma}\left(g_{v}, \phi\right)$ can be decomposed using Peter-Weyl decomposition as well. For the emerged universe to be isotropic, we require the individual quantas to be as isotropic as possible, enforces $\tilde{\sigma}\left(g_{v}, \phi\right)$ to only have support over equilateral tetrahedra [16], whose area of the four faces are equal. Since each area is determined by the associated spin $j_{i}$ of the face, we see that only the coefficients with $j_{1}=j_{2}=j_{3}=j_{4}=j$ survive in the decomposition [16]

$$
\tilde{\sigma}\left(g_{v}, \phi\right)=\sum_{j} \tilde{\sigma}_{j}(\phi) \overline{\mathcal{I}}_{\vec{m}}^{j, l_{+}} \mathcal{I}_{\vec{n}}^{j, l_{+}}(2 j+1)^{2} \prod_{l=1}^{4} D_{m_{l} n_{l}}^{j_{l}}\left(g_{l}\right),
$$


where we write $j$ for $\vec{j}=\left(j_{1}, j_{2}, j_{3}, j_{4}\right)=(j, j, j, j), \mathcal{I}_{\vec{m}}^{j, l_{+}}$is the intertwiner labeled by $\iota$, and $D_{m_{l} n_{l}}^{j_{l}}\left(g_{l}\right)$ are the Wigner matrix functions for $S U(2)$.

\section{Volume dynamics and equation of state}

The decomposition (3) indicates that the time dependence of the condensate is only encoded in $\sigma_{j}(\phi)$, hence effectively the dynamics of the condensate can be given by the following action

$$
S(\overline{\tilde{\sigma}}, \tilde{\sigma})=\int \mathrm{d} \phi_{0}\left\{\sum_{j}\left[\overline{\tilde{\sigma}}_{j}\left(\phi_{0}\right) \tilde{\sigma}_{j}^{\prime \prime}\left(\phi_{0}\right)-2 \mathrm{i} \tilde{\pi}_{0} \overline{\tilde{\sigma}}_{j}\left(\phi_{0}\right) \tilde{\sigma}_{j}^{\prime}\left(\phi_{0}\right)-\xi_{j}^{2} \overline{\tilde{\sigma}}_{j}\left(\phi_{0}\right) \tilde{\sigma}_{j}\left(\phi_{0}\right)\right]+U(\overline{\tilde{\sigma}}, \tilde{\sigma})\right\},
$$

where $\tilde{\pi}_{0}=\frac{\pi_{0}}{\varepsilon \pi_{0}^{2}-1}, \xi_{j}$ is an effective parameter encodes the details of the kinetic term of the fundamental GFT action (in the isotropic restriction), and ' denote derivatives with respect to $\phi_{0}$. Finally, from a rather phenomenological approach, the interaction kernel $U(\tilde{\sigma}, \tilde{\sigma})$ can be modelled in a simple, rather general form [24]:

$$
U(\overline{\tilde{\sigma}}, \tilde{\sigma})=\sum_{j}\left(\frac{2 \lambda_{j}}{n_{j}}\left|\tilde{\sigma}_{j}\left(\phi_{0}\right)\right|^{n_{j}}+\frac{2 \mu_{j}}{n_{j}^{\prime}}\left|\tilde{\sigma}_{j}\left(\phi_{0}\right)\right|^{n_{j}^{\prime}}\right)
$$

where $\lambda_{j}$ and $\mu_{j}$ are interaction couplings correspond to each mode $j$ satisfy that $\left|\mu_{j}\right| \ll$ $\left|\lambda_{j}\right| \ll\left|\xi_{j}^{2}-\tilde{\pi}_{0}^{2}\right|$ and we assume that constants $n_{j}^{\prime}>n_{j}>2$.

Varying the action (4) with respect to $\overline{\tilde{\sigma}}_{j}$ we can get the equation of motion [22,24]. For the purpose that will be clear later, we can decompose $\tilde{\sigma}_{j}(\phi)=\rho_{j}(\phi) \exp \left[\mathrm{i} \theta_{j}(\phi)\right]$ into module $\rho_{j}(\phi)$ and phase $\theta_{j}(\phi)$, then the equation of motion splits into imaginary and real part respectively. The imaginary part corresponds to a conserved quantity

$$
Q_{j}=\left(\theta_{j}^{\prime}-\tilde{\pi}_{0}\right) \rho_{j}^{2}
$$

whose derivative vanishes, $Q_{j}^{\prime}=0$. Then the real part becomes $[22,24]$

$$
\rho_{j}^{\prime \prime}-\frac{Q_{j}^{2}}{\rho_{j}^{3}}-m_{j}^{2} \rho_{j}+\lambda_{j} \rho_{j}^{n_{j}-1}+\mu_{j} \rho_{j}^{n_{j}^{\prime}-1}=0,
$$

where $m_{j}^{2}=\xi_{j}^{2}-\tilde{\pi}_{0}^{2}$. This equation can be integrated once directly, provides another conserved quantity,which corresponds to "clock-time translation" invariance of the system $[16,22,24]$,

$$
E_{j}=\frac{1}{2}\left(\rho_{j}^{\prime}\right)^{2}+\frac{Q_{j}^{2}}{2 \rho_{j}^{2}}-\frac{1}{2} m_{j}^{2} \rho_{j}^{2}+\frac{\lambda_{j}}{n_{j}} \rho_{j}^{n_{j}}+\frac{\mu_{j}}{n_{j}^{\prime}} \rho_{j}^{n_{j}^{\prime}} .
$$

Now we are in a position to extract observables from the condensates. In the condensate, for each equilateral tetrahedra characterized by mode $j$ we can associate a volume $\mathcal{V}_{j} \propto l_{p}^{3} j^{3 / 2}$, and the module square $\left|\sigma_{j}\right|^{2}=\rho_{j}^{2}$ gives the number of such tetrahedra. Hence the total volume of the universe can be approximated by (ignoring the quantum fluctuations) $[16,22]$

$$
\mathcal{V}\left(\phi_{0}\right) \approx \sum_{j} \mathcal{V}_{j} \rho_{j}\left(\phi_{0}\right)^{2}
$$


Using equations (7) and (8), we get the modified FLRW equations [16]

$$
\begin{aligned}
\left(\frac{\mathcal{V}^{\prime}}{3 \mathcal{V}}\right)^{2} & =\left[\frac{2 \sum_{j} \mathcal{V}_{j} \sqrt{2 E_{j} \rho_{j}^{2}-Q_{j}^{2}+m_{j}^{2} \rho_{j}^{4}-\frac{2}{n_{j}} \lambda_{j} \rho_{j}^{n_{j}+2}-\frac{2}{n_{j}^{\prime}} \mu_{j} \rho_{j}^{n_{j}^{\prime}+2}}}{3 \sum_{k} \mathcal{V}_{k} \rho_{k}^{2}}\right]^{2}, \\
\frac{\mathcal{V}^{\prime \prime}}{\mathcal{V}} & =\frac{2 \sum_{j} \mathcal{V}_{j}\left[2 E_{j}+2 m_{j}^{2} \rho_{j}^{2}-\left(1+\frac{2}{n_{j}}\right) \lambda_{j} \rho_{j}^{n_{j}}-\left(1+\frac{2}{n_{j}^{\prime}}\right) \mu_{j} \rho_{j}^{\prime}\right]}{\sum_{k} \mathcal{V}_{k} \rho_{k}^{2}} .
\end{aligned}
$$

In the free case where $\lambda_{j}=\mu_{j}=0$, the usual FLRW equation can be reproduced when the total volume is large [16].

In a homogeneous and isotropic universe, the modified FLRW equations (10) and (11) are enough enough to track the evolution of our universe. However, the relevant features of the dynamics can be extracted more easily from a deduced quantity, the effective equation of state $w=-1-2 \dot{H} /\left(3 H^{2}\right)$, where $H$ is the Hubble parameter and the dot represents derivative respect to the co-moving time $t$ [3]. In the relational language, $w$ can be rewritten as [10]

$$
w=3-\frac{2 \mathcal{V} \mathcal{V}^{\prime \prime}}{\left(\mathcal{V}^{\prime}\right)^{2}}
$$

where $\mathcal{V}$ is the total volume and the ' indicates the derivative with respect to the relational time $\phi$, and we choose the time gauge, in which the volume $\mathcal{V}=a^{3}$ for scale factor $a$.

In the following we will discuss the late time cosmological expansion using the effective equation of state (12).

\section{Late time acceleration of the universe}

In general, there is an early time acceleration phase in our model, dominated by free parameters of the condensate, but such phase ends quickly, no matter we consider only a single mode [23] or multiple modes [10]. When including interactions, however, the phase of late time acceleration will emerge and could be long lasting. In particular, with two interactions it's possible to combine inflation and late time acceleration phase together [10], but for simplicity in this report we only consider the case where each mode has only one interaction term and setting $\mu_{j}=0$ in equations (7) and (8) for example.

When $\rho_{j}$ is large, since $n_{j}>2$ and $\mu_{j}=0$ we see that the $\lambda$ term dominates in equation (8), which can be approximated to

$$
\rho_{j}^{\prime}(\phi)=\sqrt{\frac{-2 \lambda_{j}}{n_{j}}} \rho\left(\phi_{j}\right)^{\frac{n_{j}}{2}} .
$$

We require $\lambda_{j}<0$ so that $\rho_{j}^{\prime}$ stays real. Equation (13) can be easily solved and we obtain

$$
\rho_{j}(\phi)=\left(\frac{2}{n_{j}-2} \sqrt{\frac{-2 \lambda_{j}}{n_{j}}}\right)^{-\frac{2}{n_{j}-2}} \frac{1}{\left(\phi_{j \infty}-\phi\right)^{\frac{2}{n_{j}-2}}},
$$

where $\phi_{j \infty}$ is a constant of integration, determined by initial conditions and we have approximately [10]

$$
\phi_{j \infty}=-\frac{\ln \left[-\lambda_{j} /\left(2 m_{j}^{2}\right)\right]}{\left(n_{j}-2\right) m_{j}}+\frac{1}{2 m_{j}} \ln \left[\frac{n_{j}^{\frac{2}{n_{j}-2}}\left(2 m_{j}^{2}\right)}{\sqrt{E_{j}^{2}+m_{j}^{2} Q_{j}^{2}}}\right] .
$$


Since for each $j$ the module $\rho_{j}$ will diverge at $\phi=\phi_{j \infty}$, the total volume $\mathcal{V}=\sum_{j} V_{j} \rho_{j}^{2}$ will be dominated by the mode with smallest $\phi_{j \infty}$. Note that the divergence of volume at finite relational time won't lead to singularities in our model, as the effective energy density of the whole universe remains finite [10].

On the other hand, other modes, even sub-dominated, can still modify the universe evolution. In fact, adding another mode will change the way of how the effective equation of state $w$ approaches its asymptotic value [10]. To see this, we consider the case with two modes, and to save space, we use $\rho_{1,2}$ to represent $\rho_{j_{1}, j_{2}}$ and similarly for other parameters. Keeping in mind that in this report we set $\mu_{1}=\mu_{2}=0$, then at large volume $w$ will be dominated by the $\lambda$ terms as well as the volume. For simplicity we further assume that $n_{1}=n_{2}=n$, then $w$ will only depend on the ratio $r=\rho_{2} / \rho_{1}$ in the large volume limit

$$
\begin{aligned}
w & =3-\frac{(2+n)\left(\mathcal{V}_{1}+r^{2} \mathcal{V}_{2}\right)\left(\mathcal{V}_{1} \lambda_{1}+r^{n} \mathcal{V}_{2} \lambda_{2}\right)}{2\left(\mathcal{V}_{1}^{2} \lambda_{1}+r^{2+n} \mathcal{V}_{2}^{2} \lambda_{2}-2 r^{1+\frac{n}{2}} \mathcal{V}_{1} \mathcal{V}_{2} \sqrt{\lambda_{1} \lambda_{2}}\right)} \\
& =2-\frac{n}{2}-\left(\frac{n}{2}+1\right) \frac{\mathcal{V}_{1} \mathcal{V}_{2} r^{2}\left(r^{n / 2-1}-\sqrt{\lambda_{1} / \lambda_{2}}\right)^{2}}{\left(\sqrt{\lambda_{1} / \lambda_{2}} \mathcal{V}_{1}+\mathcal{V}_{2} r^{n / 2+1}\right)^{2}}
\end{aligned}
$$

which approaches to the asymptotic value $w \rightarrow 2-n / 2$ when the universe volume is large.

When $\phi_{1 \infty}=\phi_{2 \infty}$ the ratio $r=\frac{\rho_{2}}{\rho_{1}}=\left(\frac{\lambda_{1}}{\lambda_{2}}\right)^{\frac{n}{4}-\frac{1}{2}}$ becomes a constant. Submitting to equation (16) we get a constant equation of state $w=2-n / 2$. And further analysis shows that $w$ approaches this asymptotic value from above [10]. While for $\phi_{1 \infty}<\phi_{2 \infty}$, $\rho_{1}$ diverges before $\rho_{2}$ does, hence $r \rightarrow 0$ and $w$ will approach the asymptotic value from below.

The case with $n=6$ is of particular interest since $w=-1$ corresponds to the equation of state of a cosmological constant. Without losing generality we can assume $\phi_{1 \infty}<\phi_{2 \infty}$, then equation of state (16) can be expanded with respect to small $r$, and to the next to leading order we get

$$
w=-1-\frac{4 \mathcal{V}_{2}}{\mathcal{V}_{1}} r^{2}=-1-\frac{4 \mathcal{V}_{2}}{\mathcal{V}_{1}} \frac{\rho_{2}(\phi)^{2}}{\rho_{1}(\phi)^{2}}
$$

Therefore, $w<-1$ for $\phi<\phi_{1 \infty}$ and corresponds to some kind of phantom energy, whose energy density increases when the universe grows $[3,27,28]$. This leads us to the so called phantom analogue of de Sitter spacetime [29].

A further approximation can be made by noting that at large volume we can replace $\phi$ by $\phi_{1 \infty}$ in $\rho_{2}(\phi)$,

$$
\rho_{2}(\phi) \rightarrow \rho_{2}\left(\phi_{1 \infty}\right)=\left(\frac{1}{2} \sqrt{\frac{-\lambda_{2}}{3}}\right)^{-\frac{1}{2}} \frac{1}{\left(\phi_{2 \infty}-\phi_{1 \infty}\right)^{\frac{1}{2}}}
$$

Furthermore, since $\rho_{1}(\phi) \rightarrow \infty$, the contribution to total volume from $\rho_{2}$ can be ignored, then the total volume reduces to $\mathcal{V} \approx \mathcal{V}_{1} \rho_{1}^{2}$ and we obtain

$$
w=-1-\frac{b}{\mathcal{V}}
$$

where $b=4 \mathcal{V}_{2} \rho_{2}^{2}\left(\phi_{1 \infty}\right)$ is a constant. Notice again that since $b>0$, we have $w<-1$, and the phantom divide $w=-1$ is being crossed. Furthermore, as volume grows, $w$ approaches to $w=-1$ fast enough, the effective energy density of the universe will remain finite, hence in our model there are no singularities but a de Sitter spacetime [10]. 
Figure 1 shows the evolution of $w$ in our model [10]. It's clear that in the early universe, when the volume is very small, $w<-1 / 3$, corresponds to an early acceleration phase. As the volume grows, for $\phi_{1 \infty}=\phi_{2 \infty}, w$ decreases when interactions start to dominate and approaches $w=-1$ from above, while for $\phi_{1 \infty}<\phi_{2 \infty}$ the equation of state $w$ will decreases till it crosses the phantom divide $w=-1$ and then start to increase, reaches its asymptotic value from below. This confirms our analysis in this section.

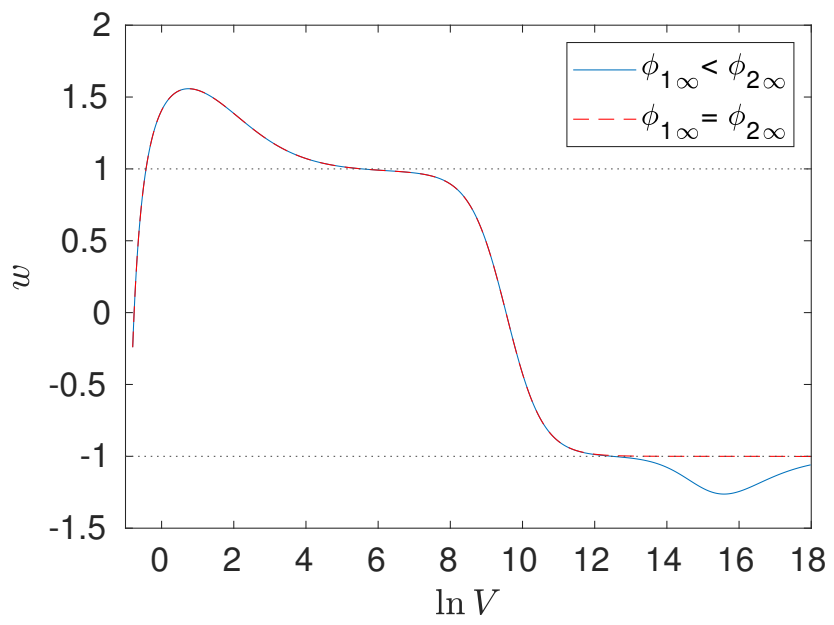

Figure 1. The behaviour of $w$ in the two modes case, where both modes have only one interaction term. Blue solid line shows the case where $\phi_{1 \infty}<\phi_{2 \infty}$, while for red dashed line we have $\phi_{1 \infty}=\phi_{2 \infty}$. Two black dotted lines show $w=1$ and the phantom divide $w=-1$, respectively. Parameters are $\mathcal{V}_{1}=1 / 3, m_{1}^{2}=3, E_{1}=5, Q_{1}^{2}=9, \lambda_{1}=-10^{-8}, \mu_{1}=0, \mathcal{V}_{2}=1 / 2, m_{2}^{2}=$ 2, $E_{2}=9, Q_{2}^{2}=2.25, \mu_{2}=0, n_{1}=n_{2}=6$, and $\lambda_{2}=-9.5 \times 10^{-8}$ for $\phi_{1 \infty}<\phi_{2 \infty}$, while $\lambda_{2}=-9.5725 \times 10^{-8}$ for $\phi_{1 \infty}=\phi_{2 \infty}$.

\section{Discussion}

This report discusses the possibility to reproduce the late time acceleration phase in the universe expansion from a candidate theory of quantum gravity, the group field theory. In this formalism, the universe is constituted by a large number of building blocks, which are excitations of the GFT field. Taking into account the homogeneity, the universe at a given time can be model by CPS, the condensate state peaked around a relational time $\phi_{0}$. The observables, such as the total volume, can be extracted from the CPS, and in particular the effective equation of state $w$ can be constructed from the volume and its derivatives.

To get the dynamics, we first show that the wave function $\sigma\left(g_{v}, \phi\right)$ can be decomposed into different modes $\sigma_{j}(\phi)$, and the evolution of the universe can be extracted by considering single or multiple modes. With a suitable choice of the effective action, the equation of motion for the module $\rho_{j}(\phi)=\left|\sigma_{j}(\phi)\right|$ can be solved approximately at large volume. We then use the solution (14) to investigate the behaviour of $w$ and find that in the two modes case with interaction of order 6, at large volume $w$ will first decreases, crosses the phantom divide $w=-1$, and then increases, getting close to its asymptotic value $w=-1$ from below. Furthermore, in our model $w$ approaches to $w=-1$ fast enough, hence we will get a de Sitter spacetime asymptotically without encountering singularities.

Acknowledgments: The author thanks China Scholarship Council for financial support.

..- Conflicts of Interest: The author declares no conflict of interest. 
1. Perlmutter, S.; Aldering, G.; Goldhaber, G.; Knop, R.A.; Nugent, P.; Castro, P.G.; Deustua, S.; Fabbro, S.; Goobar, A.; Groom, D.E.; et al. Measurements of Omega and Lambda from 42 High-Redshift Supernovae. Astrophys. J. 1998, 517, 565-586, [arXiv:astro-ph/9812133v1]. doi:10.1086/307221.

2. Riess, A.G.; Filippenko, A.V.; Challis, P.; Clocchiatti, A.; Diercks, A.; Garnavich, P.M.; Gilliland, R.L.; Hogan, C.J.; Jha, S.; Kirshner, R.P.; et al. Observational evidence from supernovae for an accelerating universe and a cosmological constant. Astron.J. 1998, 116, 1009-1038, [arXiv:astro-ph/9805201]. doi:10.1086/300499.

3. Tsujikawa, S. Dark energy: investigation and modeling 2010. pp. 331-402, [1004.1493]. doi: 10.1007/978-90-481-8685-3_8.

4. Weinberg, S. Cosmology; Oxford University Press, 2008.

5. Spergel, D.N.; Bean, R.; Doré, O.; Nolta, M.R.; Bennett, C.L.; Dunkley, J.; Hinshaw, G.; Jarosik, N.; Komatsu, E.; Page, L.; et al. Wilkinson Microwave Anisotropy Probe (WMAP) Three Year Results: Implications for Cosmology. Astrophys. J. Suppl. Ser. 2006, 170, 377-408, [arXiv:astro-ph/0603449v2]. doi:10.1086/513700.

6. Sakharov, A.D. Vacuum quantum fluctuations in curved space and the theory of gravitation. Sov.Phys.Dokl. 1968, 12, 1040-1041. doi:10.1070/PU1991V034N05ABEH002498.

7. Weinberg, S. The cosmological constant problem. Rev. Mod. Phys. 1989, 61, 1-23. doi: 10.1103/REVMODPHYS.61.1.

8. Anagnostopoulos, F.K.; Basilakos, S.; Kofinas, G.; Zarikas, V. Constraining the Asymptotically Safe cosmology: Cosmic acceleration without dark energy. JCAP 2019, 2019, [1806.10580]. doi:10.1088/1475-7516/2019/02/053.

9. Dvali, G.; Gabadadze, G.; Porrati, M. 4D Gravity on a Brane in 5D Minkowski Space. Phys. Lett. Sect. B Nucl. Elem. Part. High-Energy Phys. 2000, 485, 208-214, [arXiv:hep-th/0005016v2]. doi:10.1016/S0370-2693(00)00669-9.

10. Oriti, D.; Pang, X. Phantom-like dark energy from quantum gravity. JCAP 2021, 2021, 040, [2105.03751]. doi:10.1088/1475-7516/2021/12/040.

11. Samart, D.; Gumjudpai, B. Phantom field dynamics in loop quantum cosmology. Phys. Rev. D 2007, 76, [0704.3414]. doi:10.1103/PhysRevD.76.043514.

12. Oriti, D. The microscopic dynamics of quantum space as a group field theory 2011. [1110.5606].

13. Krajewski, T. Group Field Theories. Proc. 3rd Quantum Gravity Quantum Geom. Sch. PoS(QGQGS 2011); Sissa Medialab: Trieste, Italy, 2013; p. 005. doi:10.22323/1.140.0005.

14. Carrozza, S. Flowing in Group Field Theory Space: a Review. SIGMA 2016, [1603.01902]. doi:10.3842/SIGMA.2016.070

15. Gielen, S.; Oriti, D.; Sindoni, L. Homogeneous cosmologies as group field theory condensates. JHEP 2014, 06, 13, [arXiv:gr-qc/1311.1238]. doi:10.1007/JHEP06(2014)013.

16. Oriti, D.; Sindoni, L.; Wilson-Ewing, E. Emergent Friedmann dynamics with a quantum bounce from quantum gravity condensates. Class. Quant. Grav. 2016, 33, 224001, [arXiv:grqc/1602.05881]. doi:10.1088/0264-9381/33/22/224001.

17. Gielen, S.; Sindoni, L. Quantum Cosmology from Group Field Theory Condensates: a Review. SIGMA 2016, [1602.08104]. doi:10.3842/SIGMA.2016.082.

18. Oriti, D. The universe as a quantum gravity condensate. Comptes Rendus Phys. 2017, 18, 235-245, [arXiv:gr-qc/1612.09521]. doi:10.1016/j.crhy.2017.02.003.

19. Pithis, A.G.; Sakellariadou, M. Group field theory condensate cosmology: An appetizer, 2019, [1904.00598]. doi:10.3390/universe5060147.

20. Gielen, S.; Polaczek, A. Generalised effective cosmology from group field theory. Class. Quant. Grav. 2019, 37, [1912.06143]. doi:10.1088/1361-6382/ab8f67.

21. Marchetti, L.; Oriti, D. Quantum fluctuations in the effective relational GFT cosmology 2020. [2010.09700].

22. Marchetti, L.; Oriti, D. Effective relational cosmological dynamics from Quantum Gravity 2020. [2008.02774].

23. de Cesare, M.; Sakellariadou, M. Accelerated expansion of the Universe without an inflaton and resolution of the initial singularity from Group Field Theory condensates. Phys. Lett. B 2017, 764, 49-53. doi:10.1016/j.physletb.2016.10.051.

24. de Cesare, M.; Pithis, A.G.A.; Sakellariadou, M. Cosmological implications of interacting Group Field Theory models: cyclic Universe and accelerated expansion. Phys. Rev. 2016, D94, 64051, [arXiv:gr-qc/1606.00352]. doi:10.1103/PhysRevD.94.064051. 
25. Oriti, D. Group field theory as the 2nd quantization of Loop Quantum Gravity. Class. Quant. Grav. 2016, 33, 85005, [arXiv:gr-qc/1310.7786]. doi:10.1088/0264-9381/33/8/085005.

26. Rovelli, C. Quantum gravity; Cambridge university press, 2004.

27. Caldwell, R.R.; Kamionkowski, M.; Weinberg, N.N. Phantom Energy and Cosmic Doomsday. Phys. Rev. Lett. 2003, 91, [arXiv:astro-ph/0302506]. doi:10.1103/PhysRevLett.91.071301.

28. McInnes, B. What If $\mathrm{w}<-1$ ? 2002. [arXiv:astro-ph/0210321].

29. McInnes, B. The dS/CFT Correspondence and the Big Smash. JHEP 2001, 6, 615-637, [arXiv:hep-th/0112066]. doi:10.1088/1126-6708/2002/08/029. 
